\title{
Anabases
}

ANABASES Traditions et réceptions de l'Antiquité

4 | 2006

Varia

\section{Geoffroy Tory et les leçons de l'Antique}

\section{Pierre Cordier}

\section{OpenEdition}

Journals

Édition électronique

URL : http://journals.openedition.org/anabases/3551

DOI : 10.4000/anabases.3551

ISSN : 2256-9421

\section{Éditeur}

E.R.A.S.M.E.

\section{Édition imprimée}

Date de publication : 1 octobre 2006

Pagination : 11-32

ISSN : 1774-4296

\section{Référence électronique}

Pierre Cordier, "Geoffroy Tory et les leçons de l'Antique », Anabases [En ligne], 4 | 2006, mis en ligne le 01 mai 2012, consulté le 21 octobre 2019. URL : http://journals.openedition.org/anabases/3551 DOI : 10.4000/anabases.3551 
Anabases 4 (2006), p. 11-32

\section{Geoffroy Tory et les leçons de l'Antique}

PIERRE CORDIER

$\phi(\lambda T a ́ T \omega$

Jean Letrouit.

GEoffroy Tory, né à Bourges vers 1480, dans une famille modeste, et formé dans sa ville natale, visita l'Italie, où il étudia, à Rome, avec Béroalde, puis à Bologne, en 15051506 1. À son retour en France, il devint correcteur chez Gilles de Gourmont, dans l'atelier de qui il réalisa en 1507 sa première édition, un Pomponius Mela, publié en 1508 avec un envoi à Philibert Babou, valet de chambre de Louis XII, originaire de Bourges comme lui. Sous la protection de ce compatriote, il devint, à Paris, régent (professeur) au collège du Plessis (1509), puis au collège Coqueret (1511), enfin professeur de philosophie au collège de Bourgogne. Parallèlement, Geoffroy Tory poursuivait une activité éditoriale (il publia en 1512 le traité De re aedificatoria d'Alberti) et se formait à la gravure de caractères auprès du peintre Jean Perréal ${ }^{2}$. Ses finances ayant eu

1 Sur le voyage en Italie, $c f$. Champ Fleury, fo XLIX a. Sur Tory, l'ouvrage fondamental reste l'étude d'Auguste J. BERNARD, Geoffroy Tory, peintre et graveur, premier imprimeur royal, réformateur de l'orthographe et de la typographie sous François Ier, 2e éd. refondue, Paris, Tross, 1865 (réimpr. Nieuwkoop, B. de Graaf, 1963) ; cf. aussi Ph. Goldman, "Geoffroy Tory et Bourges", in Actes du $55^{\circ}$ Congrès de la fédération des sociétés savantes du centre de la France (Limoges, 17-19 mai 1996) = Bulletin de la société archéologique et historique du Limousin 125 (1997), p. 44-61.

2 Leonis Baptistae Alberti Florentini... Libri de re aedificatoria decem. Opus integrum et absolutum; diligenterque recognitum... Facta est etiam capitum ipsorum non inelegans tabula..., Paris, 1512, dédicacé à Ph. Babou et J. Lallemand. Jean Perréal fournit un dessin au Champ fleury (fo XLVI b). 
à souffrir d'un second voyage en Italie (1516-1517), au cours duquel il étudia les antiques, Tory s'établit en 1518, à son retour à Paris, comme enlumineur et graveur. Cette activité lui assura quelque notoriété : il illustra dans les années 1520 , en collaboration avec Simon de Collines, des livres d'heures ${ }^{3}$ qui lui attirèrent la bienveillance du roi François Ier. En 1533, Tory fut nommé imprimeur du roi, puis libraire juré de la Sorbonne. Son activité est d'une grande diversité : outre les Livres d'Heures évoqués plus haut, il produisit des éditions annotées d'auteurs anciens (Pomponius Mela en 1508, Bérose de 1509 à 1511, Probus en 1510, Quintilien en 1511), il traduisit l'Économique de Xénophon ${ }^{4}$, il édita dans les années 1530 des traductions en français des versions latines de Plutarque, de Lucien, de Diodore (traduction d'Antoine Macault) et d'Eusèbe de Césarée (traduction de Claude de Seyssel) ${ }^{5}$, des ouvrages savants composés en Italie (le De re aedificatoria d'Alberti en 1512, les Chroniques de Jules César à Maximilien d'Egnasius en 1529). À mesure qu'il gagnait la faveur royale et consolidait sa position, il édita enfin plusieurs commandes d'ouvrages de circonstance sur le sacre de la reine, l'adventus du souverain et l'éloge funèbre de la mère du roi. Une fois imprimeur royal, il publia les ordonnances officielles, mais aussi l'Adolescence clementine de

3 Horae in laudem beatissimae semper Virginis Mariae secundum consuetudinem curiae Romanae, Paris, 1524[-1525]; Heures à la louange de la vierge Marie selon lusage de Rome... le tout au long... est tres correct en bone orthographie..., Paris, 1525 ; [Nouvelle éd.] Horae in laudem beatiss[imae] Virg[inis] Mariae ad usum Romanum venales extant Parrhisiis ad insigne vasis effracti, Paris, 1527. Dans les éditions postérieures, publiées à Paris, le nom de la capitale est orthographié Parrhisiis: Hore in laudem sanctissime virginis Marie secundum consuetudinem ecclesiae Parisiensis, 1527 ; Horae in laude[m] beatissimae Virginis Mariae, secundum usum Romanum, 1529 ; Horae in laudem beatiss[imae] virginis Mariae. Ad usum Romanum, 1531.

Science pour senrichir honnestement et facilement, intitulee Leconomic Xenophon, nagueres translatee de grec et latin en langaige francoys par maistre Geofroy Tory de Bourges, Paris [1530].

5 Politiques de Plutarche, cest a dire : Civiles institutions et enseignements pour bien regir la chose pu[blique], jadis composees en grec par Plutarche, et depuis translatees de grec en latin par le seigneur Nicole Sagundin, et a present de langue grecque et latine en langaige francois par maistre Geofroy Tory de Bourges. Dediees par ledit autheur a lempereur Trajan, et par le translateur en langaige francois a tresilustre et plain de bon espoir en toute heureuse vertu, son seigneur, François de Vallois, Dauphin de France, Paris, par maistre Geofroy Tory de Bourges, [1532] ; La Mouche de Lucian, et la maniere de parler et de se taire [de Volaterran]. La mousche est translatée de latin et de grec en langaige françois ; la maniere de parler et de se taire est translatee seullement de latin en françois. Le tout par maistre Geofroy Tory de Bourges, imprimeur du Roy et libraire juré en luniversité de Paris, Paris, [1533] ; Les troys premiers livres de lhistoire de Diodore Sicilen, historiographe grec... translatez de latin en francoys par maistre Anthoine Macault..., Paris, 1536 (édition posthume probablement achevée à l'instigation de son épouse); Lhistoire ecclesiastique (d'Eusèbe) translatee de latin en francois par messire Claude de Seyssel, evesque lors de Marseille, depuis archevesque de Thurin. Imprimee par le commandement du Roy, Paris, 1532. 
Clément Marot ${ }^{6}$. Le Champ fleury marqua un tournant dans l'activité de Tory : après 1529, il se lança dans l'impression d'ouvrages de plus en plus soignés, destinés à un public prestigieux, tout en prêchant pour l'adoption de règles orthographiques, comme le souligne le titre de l'Adolescence clementine 7 . Parallèlement, il fit éditer des compositions personnelles, notamment une élégie sur la mort de sa fille survenue en $1522^{8}$. Le catalogue des œuvres écrites ou publiées par Tory donne en somme une bonne idée du foisonnement, mélange d'érudition, d'innovation et de littérature de combat, qui caractérise son Champ fleury.

En dépit de son apparent décousu, des digressions et des bigarrures introduites dans le texte par les références érudites et les prises de position dans les débats du temps, le Champ fleury de Geoffroy Tory (1529) ${ }^{9}$ montre une profonde et subtile unité de

6 Le sacre et le coronnement de la Royne, imprime par le commandement du Roy nostre Sire, Paris, 1531 ; Lentree de la Royne en sa ville et cite de Paris, imprimee par le commandement $\mathrm{du}$ Roy nostre Sire [Paris, 1531], in Lodoicae regis matris mortem epitaphia latina et gallica. Epitaphes a la louenge de ma dame mere du roy faictz par plusieurs recommandables autheurs, Paris, 1531 ; Ladolescence clementine. Autrement les xuvres de Clement Marot de Cahors en Quercy... composees en leage de son adolescence. Avec la complaincte sur le trepas de feu messire Florimond Robertet. Et plusieurs autres auvres faictes par ledict Marot depuis leage de sa dicte adolescence, Le tout revu, corrige et mis en bon ordre, Paris, 1532.

7 (1533) : avec certains accents notez, c'est assavoir sur le é masculin different du feminin, sur les dictions ioinctes ensembles par sinalephes et souz le c quand il tient de la pronunciation de le s, ce qui par cy devant par faute daduis na este facit au langaige francoys combien q'il y fust et soyt tresnecessaire. Cf. Nina CATACH, Histoire de l'orthographe française, éd. posthume réalisée par R. Houvault, Paris, Champion (Lexica, 9), 2001, p. 106-111.

Gotofredi Torini, Biturici in filiam charissi[mam], virguncularum elegantissimam, epitaphia et dialogi. In eandem etiam quatuor et viginti disticha, unum et eundem sensum copia verborum et ingenii foecunditate pulchre repetentia, Paris, 1524. Cf. aussi l'Aediloquium, ceu Disticha, partibus aedium urbanarum et rusticarum suis quaeque locis adscribenda. Item, Epitaphia septem de amorum aliquot passionibus, antiquo more et sermone veteri, vietoque conficta, Paris, 1530.

9 Geoffroy Tory, Champ Fleury. Auquel est contenu l'art \& science de la deue et vraye proportion des lettres attiques, qu'on dit autrement lettres antiques, \& vulgairement lettres romaines proportionnées selon le corps et visage humain. Ce livre est privilegie pour dix ans par le Roy nostre Sire, \& est a vendre a Paris sus Petit Pont a lenseigne du Pot Casse par Mestre Geoffroy Tory de Bourges - libraire, et Autheur dudict livre, et par Gils Gourmont aussi libraire demeurant en la rue Sainct Jacques a lenseigne des Trois Couronnes [1529] ; Id., réédition par Olivier Mallard, 1539, puis Vincent Gautherot, Paris, 1549. Reproductions phototypiques de l'édition princeps de Paris 1529 : par Gustave CoHEN (avant-propos, notes, index et glossaire), Paris, Charles Bosse, 1931 (réimpr. Genève, Slatkine reprints, 1972) ; avec intr. de Paul-M. Grinevald, Paris, Bibliothèque de l'image, 1998 ; par Kay AMERT avec la trad. annotée de George B. Ives ( $c f$. ci-après), Oakland, Octavo, $2004(2$ CD-Rom, non vidi). Éd. angl. par John JollifE (intr.), George B. Ives (trad.), Champ Fleury, New York, Grolier Club, 1927, réimpr. Wakefield / New York / Paris, S. R. Publishers / Johnson reprints / Mouton, 1970. 
composition. Je commencerai par donner une brève présentation du Champ fleury même. Entre résumé et survol critique, elle permettra de mettre en évidence l'omniprésence et la complexité des références à l'Antiquité : Tory tire de l'Antiquité un enseignement, mais il en présente aussi une lecture orientée par un projet personnel. C'est à ce double sens du mot "leçon " - enseignement et façon de lire - que fait allusion le titre du présent article. Pour traiter la question, bien conscient d'opérer dans le vaste corpus du Champ fleury une sélection arbitraire, j'aborderai ensuite trois thèmes : le débat sur le modèle des caractères écrits, l'origine grecque des Français et de la langue française selon Tory et les résonances cosmologiques et anthropologiques de l'œuvre.

Le Champ fleury fut élaboré, pour l'essentiel, dans la seconde moitié des années 1520 (le projet initial remonte à janvier 1523 ; Tory obtint un privilège royal en 1526 et l'ouvrage parut en 1529). Geoffroy Tory, après son second voyage en Italie, cherchait la notoriété et obtint l'appui du roi. Son ascension accompagna celle de son protecteur Philibert Babou, qui devint en 1536 surintendant de tous les bâtiments du royaume. Le projet d'une défense et illustration de la langue française avant la lettre était un manifeste d'adhésion aux visées royales de promotion du français, d'émulation avec l'Italie et d'exaltation de la France, que montrait la politique de François Ier.

L'ouvrage comporte quatre parties : trois livres et un appendice formé de deux cahiers de planches, le tout précédé d'un premier envoi " a tous vrayz et deuotz Amateurs de bonnes lettres ", et d'un second envoi « aux lecteurs de ce present livre ", qui insiste notablement sur le lien entre vertu et bon langage. Le premier livre ( $\left.f^{\circ} I-X\right)$ énonce le propos de l'œuvre : « decorer » et « enluminer » la langue française à l'exemple de ce que les Anciens, Grecs et Romains, ont fait pour leur propre langue ( $\left.f^{\circ} \mathrm{I}-\mathrm{IVb}\right)$. Le français entretenant avec le grec et l'hébreu un lien de filiation directe, les Français étant eux-mêmes des Hellènes, le grec fournit un modèle plus vénérable et plus authentique que le latin pour l'introduction de normes dans le langage français. Deux développements mythiques appuient ce propos : le mythe de l'Hercule Gaulois, Ogmios, dieu national du peuple le plus éloquent et dont la puissance s'exerçait par l'attrait de la parole, faisant du dieu la version gauloise du Mercure grec ${ }^{10}$. C'est la figure de Mercure (Hermès, mais Tory lui donne son nom latin) qui relie le second récit étiologique au premier. Tory explique l'origine des lettres en recourant au mythe d'Io. Transformée en vache, elle imprime avec son sabot son nom dans le sol pour se faire reconnaitre de son père. La combinaison de ces deux caractères originels - deux formes géométriques, la ligne droite du iota et la courbe de l'omicron, que Tory, pour les besoins de la démonstration, substitue à l'omega de $I \Omega$-, donne naissance au tracé de toutes les autres lettres. Le dieu Mercure apparait dans une seconde version de l'origine des caractères, qui attribue cette invention à un récit de métamorphose : comme Apollon a assassiné Hyacynthe, ce dernier se change en une fleur frappée des lettres upsilon ( $\mathrm{Y}=$ iota accolés en fourche) et alpha (A) ( $\mathrm{f}^{\circ} \mathrm{IX} \mathrm{b}$ et XXX a, fig. 1). Outre leur 


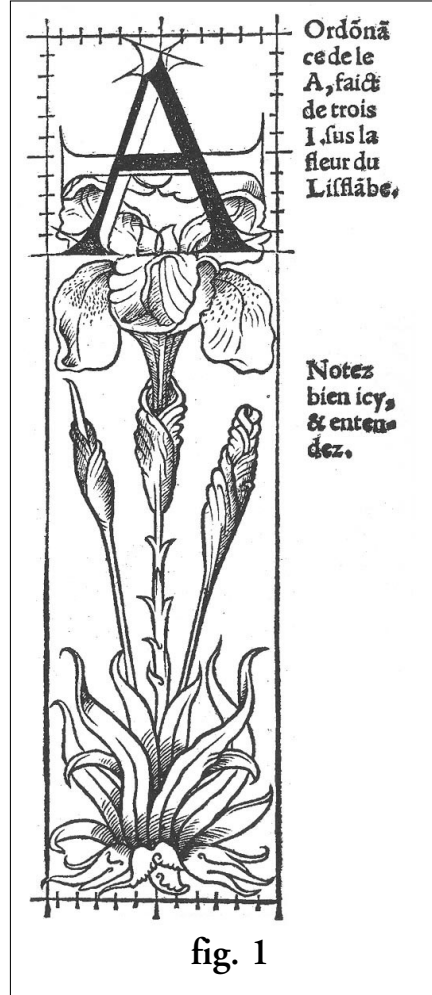

connexion par la figure de Mercure, les deux mythes sont ainsi reliés par une combinatoire géométrique (droite et cercle ou droite et triangle).

Le livre II ( $f^{\circ} \mathrm{XI}-\mathrm{XXX}$ a) s'ouvre sur les principes de la géométrie, des diagrammes illustrant les figures élémentaires - carré, ligne droite, courbe, triangle - $\left(\mathrm{f}^{\circ}\right.$ XI b-XII a) ; une digression invite à composer des traités de géométrie en français. Critiquant les modèles géométriques de ses prédécesseurs dans l'art de tracer les caractères, Tory définit une grille universelle permettant de normaliser la forme des lettres et leurs proportions : un carré divisé, verticalement, en rangs et, horizontalement, en colonnes de 10 carrés plus petits, soit un damier à 100 cases ( $f^{\circ}$ XXXIII a). La suite du livre exploite les implications mathématiques et mythologiques de ce découpage. Dans le carré décimal, toutes les figures élémentaires (droite, cercle, triangle) peuvent venir s'inscrire à proportions constantes; le I peut recevoir, en chacun des rangs horizontaux qui le compose, le nom d'Apollon et d'une Muse ; le $\mathrm{O}$, de même, embrasse le nom du dieu et celui des sept arts libéraux. Les

deux figures géométriques que sont le I et le $\mathrm{O}$ se combinent pour former la flûte du berger Corydon dans les Bucoliques de Virgile : elle est droite comme un I, sa bouche forme un $\mathrm{O}$ et ses sept trous sont les Muses ( $f^{\circ} \mathrm{XV}$ b et XVI, fig. 2 et 3$)^{11}$. Mythologique et exprimant l'architecture du savoir, le carré décimal livre également la grille sur laquelle l'Homme lui-même est bâti et orienté. La plus grande partie du livre est ensuite consacrée à développer les rapports entre corps humain, visage humain et quadrature des lettres ( $f^{\circ} \mathrm{XVII}-$ XVIII). L'homme de Vitruve est composé de dix parts de hauteur égale et les parties

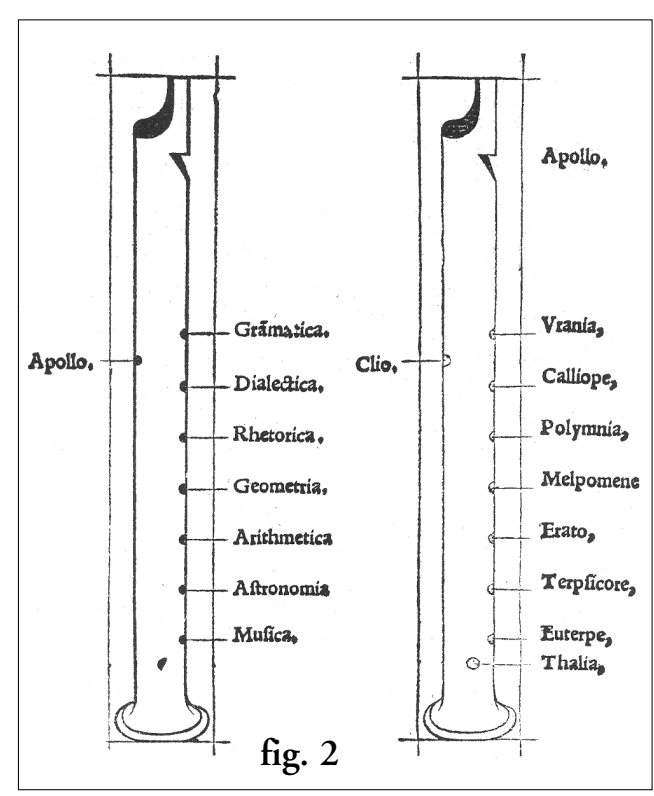

11 Bucoliques, II, 36-37 : Est mihi disparibus septem compacta cicutis / Fistula, Damoetas dono quam dedit olim. 

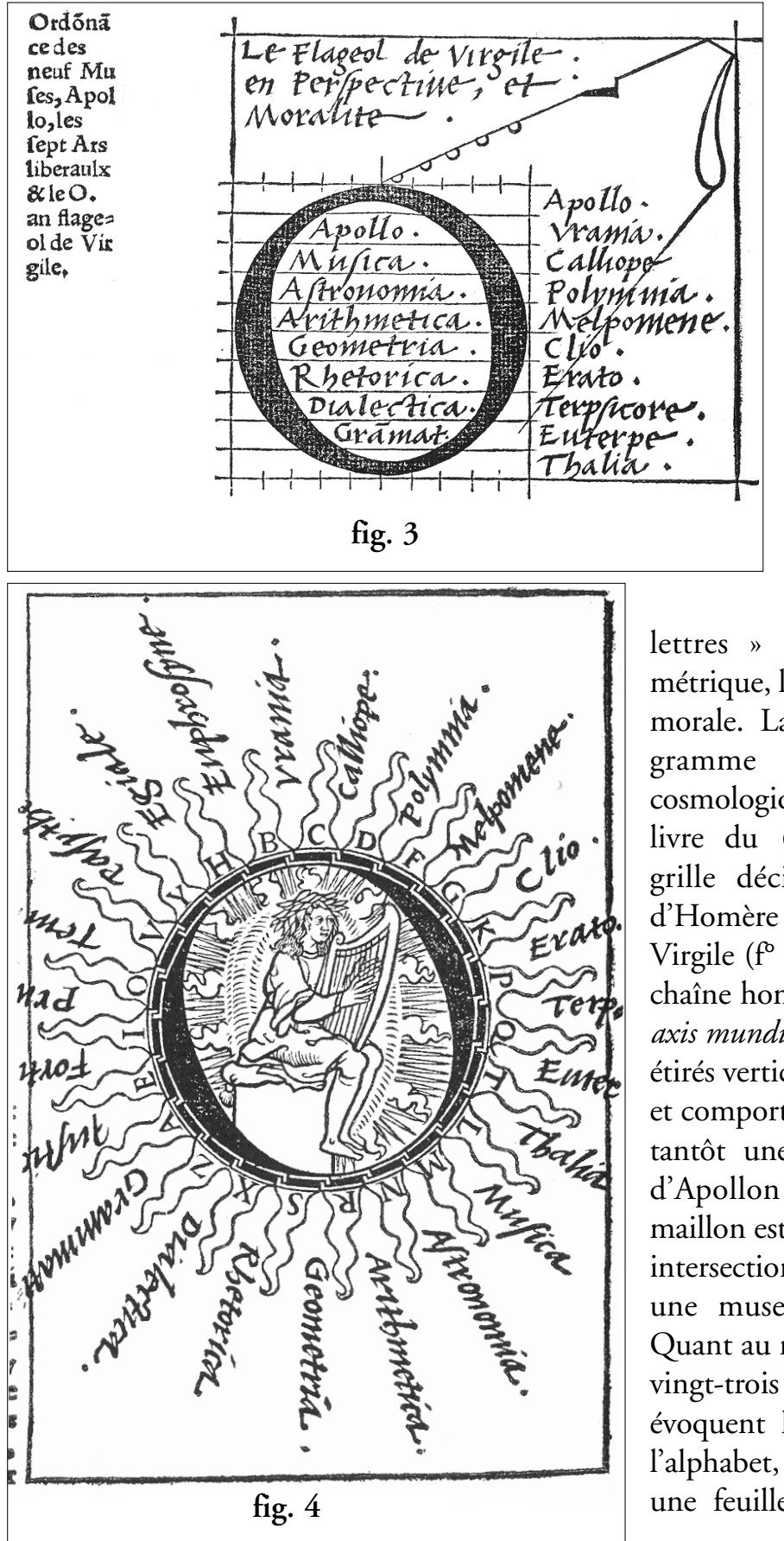

de son corps entretiennent avec les parties des lettres des correspondances géométriques : un A est bien dessiné lorsque sa barre médiane vient dissimuler les pudenda d'un homme inscrit selon les règles dans la même grille. La correspondance veut " denoter que Pudicite et Chastete auant toutes choses sont requises en ceulx qui demandent acces et entree aux bonnes lettres " ( $\left.\mathrm{f}^{\circ} \mathrm{XIX} b\right)$. Anthropométrique, la correspondance est aussi morale. La lettre devient un idéogramme anthropologique, voire cosmologique : la fin du deuxième livre du Champfleury applique la grille décimale à la chaîne d'or d'Homère et au rameau d'or de Virgile (fo XXV b-XXIX a). Dans la chaîne homérique, il voit tantôt une axis mundi, une série de 10 maillons étirés verticalement de la terre au ciel et comportant, en son milieu, un $\mathrm{O}$, tantôt une chaîne formant autour d'Apollon un $\mathrm{O}$, dont chaque maillon est une lettre et dont chaque intersection correspond à un art ou à une muse ( $f^{\circ}$ XXVIII b, fig. 4). Quant au rameau d'or de Virgile, les vingt-trois vers relatifs à l'épisode évoquent le nombre des lettres de l'alphabet, dont chacune constitue une feuille ${ }^{12}$. Le rameau compte

12 Virgile, Énéide 6, 126-148. Tory coupe le passage pour n'en retenir que vingt-trois vers. 


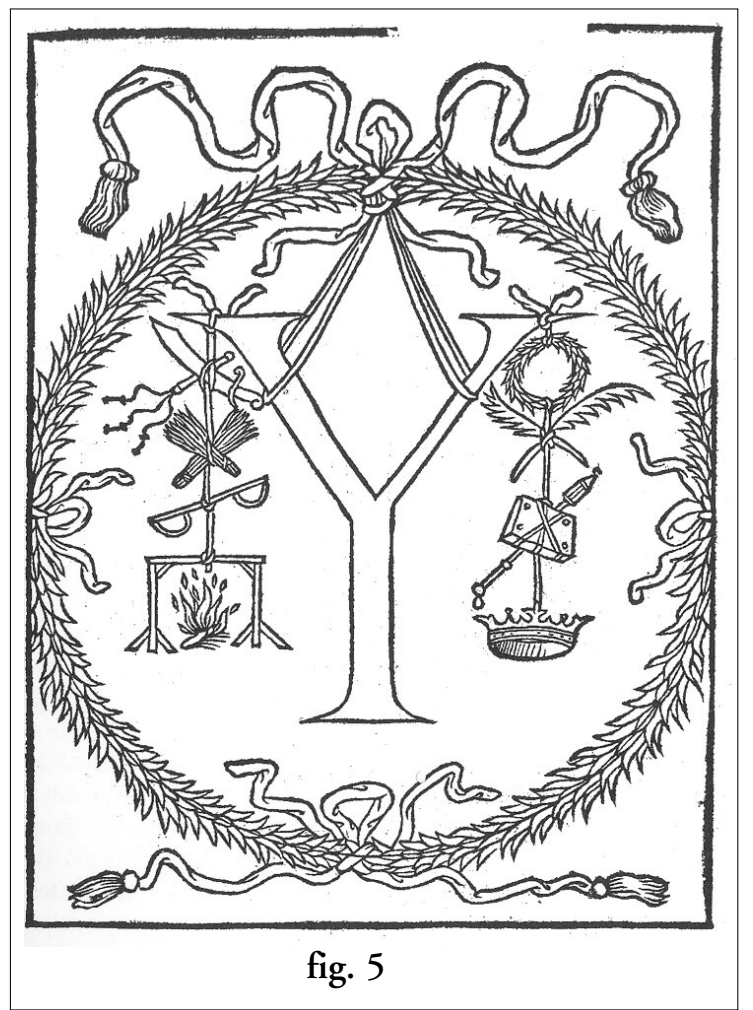

trois branches : au centre, les consonnes (BCDFGKPQT), au nombre de neuf comme les Muses, à gauche, sept semi-vocales, qui sont les arts libéraux (LMNRSXZ), à droite, sept lettres (les voyelles et le $\mathrm{H}$ aspiré « laquelle pour ce quelle nest du tout reputee uraye lettre, est escripte en la plus basse fueille ") sont les quatre vertus cardinales et " les trois Graces de uenuste et de decente honestete». Le livre s'achève sur le triomphe d'Apollon et des Muses.

Aux yeux d'un lecteur contemporain, seul le livre III relève directement du programme énoncé par le titre et présente un manuel de typographie, fournissant les instructions pour tracer chaque lettre, dans sa grille, à la règle et au compas. Fait notable, la présentation des lettres ne se limite pas à

leur seule structure graphique, mais embrasse également les problèmes posés par sa prononciation : c'est que pour Tory, la lettre est à la fois une figure géométrique, un son, un vecteur de sens et une figure de l'humain. Cette perspective est parfaitement cohérente avec l'argumentation du livre deux, que je viens de résumer. En arrivant, à la fin de l'alphabet, à la lettre Y (fo LXI b-LXIII), Geoffroy Tory fait de ce caractère une lecture allégorique et morale, invoquant Hercule au carrefour. Le dieu hésite, à la bifurcation de son chemin, entre la voie de Volupté et la voie de Vertu ${ }^{13}$. Deux emblèmes suivent ( $f^{\circ}$ LXIII a et $b$, fig. 5), où la lettre historiée illustre le dilemme moral. Le Z est un escalier à neuf marches ( $f^{\circ} \mathrm{LV}$ a) : "Ces marches et degres la, nous signifient en Sens moral, la voye, \& lascendant a beatitude, que peuvent avoir facilement ceulx qui ont la cognoissance \& perfection des bonnes lettres, Ars, \& Sciences. À propos de quoy iay designe au dessus de la lettre ung petit esperit diuin estant sus les pieds prompt ou a donner la Coronne, le sceptre, la Palme, ou le Chapeau de laurier, a tous ceulx qui bien la croisée des chemins : et autres matériaux figuratifs de l'Antiquité dans l'art plus récent, Paris, Flammarion, 1999. Cf. aussi fo LXII a et manchette. 
\& diligentement se euerturont a acquerir Science, en montant de degre en degre iusques a la perfection dicelle ou gist tout acomplicement dexcellent prix, \& glorieux honneur ». Les bonnes lettres, en somme, mènent à l'exercice de vertu, et la lettre $\mathrm{Z}$ fait écho au propos de l'envoi liminaire.

Le sous-titre du Champ fleury définit l'argument principal de l'ouvrage : " enseigner l'art et science de la vraie proportion des lettres ». L'idée d'un manuel qui définirait et divulguerait les règles de conformation de l'écriture n'est pas nouvelle. Pour composer son traité de l'art de conformer les lettres, Tory pouvait s'appuyer sur des traités d'écriture existants 14 . La partie terminale de l'ouvrage (fo LVII à LXXX), qui présente une série d'alphabets non latins ou de fantaisie, est elle-même loin de constituer une innovation : de telles planches alphabétiques circulaient au moins depuis le XIVe siècle ${ }^{15}$. Mais les références récurrentes du Champ fleury sont plus récentes et témoignent de préoccupations nouvelles, liées à la reviviscence de la capitale romaine. Tory critique en détail deux traités récents, le traité De la divine proportion de Luca Pacioli, paru à Venise en 1509 avec des illustrations de Léonard de Vinci, et les Instructions sur la manière de mesurer avec la règle et le compas, troisième livre de la Géométrie d'Albrecht Dürer publié en $1525^{16}$. Le lettrage de l'épigraphie monumen-

14 P. SAENGER, "Geoffroy Tory et la nomenclature des écritures livresques françaises au XVe siècle ”, Le Moyen Âge 32 (1977), p. 493-520 ; B.C. Bowen, “Geoffroy Tory’s Champ Fleury and Its Major Sources ”, Studies in Philology 76 (1979), p. 13-27, notamment p. 18 et n. 7 ad loc. identifie Damiano da Moile, Operina (1522) et Il modo de temperare le penne (1523) ; S. Morison, A Newly Discovered Treatise on Classic Letter Design, Printed at Parma by Damianus Moyllus c. 1480, Paris, At the Sign of Pegasus, 1927. On évoquera aussi Felice Feliciano, Alphabetum romanum, R.H. BoOTHROYD et G. MARDERSTEIG (éd.), Officina Bodoni, Vérone. 1960. Cf. B.C. Bowen, “ Geoffroy Tory's Champ Fleury and Its Major Sources ”, et Hermann ZAPF, “Typographie des caractères romains de la Renaissance ", tr. fr. par C. Briand, Ph. Hody et S. Le Claire avec mise à jour bibl. de J. André in Cahiers Gutenberg 37-38, déc. 2000, [p. 44-52], p. 46 (= "Vom Formgesetz der Renaissance Antiqua", Der Polygraph, 21, 1953, Supplément 1.11 : Satz und Schrift). Cf. Bernard de Mandeville, Livre des Merveilles du monde, datant de 1356 : à la fin de la description de chaque peuple figurait un alphabet. Le plus ancien manuscrit de cet ouvrage remonte à 1372; il avait fait l'objet d'une édition incunable en 1478 et fut réédité vers 1510. Cf. aussi l'édition princeps de l'ouvrage maintes fois réédité de Bernard de Breydenbach, Grand voyage de Jérusalem, Mayence, 1486, avec sa paraphrase française par Nicolas le Huen.

16 Sur Luca Pacioli, cf. fo XIIIa. Cf. Luca Pacioli, Divine proportion (tr. du De Divina Proportione par G. Duchesne, M. GIRAud et M.T. Sarrade), Paris, Librairie du compagnonnage, 1980 ; cf. P. SpeZiali, "Luca Pacioli et son œuvre ”, Sciences of the Renaissance (Tours, 1965), Paris, Vrin, p. 93-106 ; Albrecht Dürer, Underweysung der Messung mit dem Zirckel und RichtScheit, Nuremberg, 1525. Sur la réception de Pacioli chez Tory, cf. J. DAuphine, “De l'esthétique dans le Champ fleury de Geoffroy Tory”, in Lettere e arti nel Rinascimento : atti del X convegno internazionale, Chianciano-Pienza 20-23 luglio 1998, Florence, F. Cesati, 2000, p. 313-316. 
tale romaine en était venu à fournir un modèle d'écriture : dès 1463, un manuscrit de Felice Feliciano conservé à Vérone, l'Alphabetum Romanum, proposait des reconstructions géométriques de lettres capitales romaines. En 1517, Francesco Torniello avait publié l'Opera del modo de fara le littere maiuscole antiche, con mesura de circino e resone de penna ${ }^{17}$.

La question de la majuscule antique dans le Champ fleury s'articule ici avec celle, plus générale, du caractère romain. En 1499, Alde Manuce, qui avait déjà commandé des fontes romaines à son graveur Francesco Griffo, fit produire des capitales d'après l'antique 18. En France, l'influence des éditions aldines introduisit les caractères romains, qui devinrent, avec la division en chapitres, les manchettes marginales et les indices, l'organe de la révolution orthographique et typographique des deux premières décennies du XVIe siècle, facilitant la promotion du français comme langue de diffusion du savoir et de communication intellectuelle. C'est là un point important : l'apparition d'une nouvelle culture intellectuelle coïncide avec l'élaboration de son vecteur de diffusion. Paraît ainsi, en 1522, le Summaire ou Epitome du livre De asse, résumé en français du fameux ouvrage latin de Guillaume Budé paru en 1515, deuxième ouvrage publié en France en caractères romains. Ce sont ces mêmes caractères romains, encore jeunes, qui se retrouvent dans le Champ Fleury de Geoffroy Tory, traité en français sur la composition des lettres typographiques.

Dans ces conditions, le fait que les consignes délivrées par Tory ne s'appliquent, dans le corps de l'ouvrage, qu'aux capitales, et non aux lettres de bas de casse, pourrait surprendre. Si Tory, promoteur de la langue française, utilise les minuscules romaines pour composer son ouvrage, mais se consacre à un traité de la lettre capitale, c'est parce que cette dernière est antique, à la différence du bas de casse, qui est issu, lui, d'un modèle moderne, l'écriture manuscrite humanistique. Pour justifier la normalisation de la langue, l'autorité des Anciens est efficace, notamment parce que la capitale aldine, inspirée du modèle épigraphique romain, se prête aisément à l'application de règles de proportionnalité et à l'inscription du caractère dans la grille décimale.

Le développement de Tory sur les lettres capitales réserve au lecteur une surprise : au lieu de leur reconnaître le nom de " romaines ", ou de les qualifier, à l'instar de quelques autres auteurs de traités sur la conformation des lettres, d' " antiques ", l'auteur du Champ fleury conteste l'une et l'autre appellation, pour en proposer une troisième. Ses lettres capitales sont des caractères attiques. Le titre de l'ouvrage attirait déjà

17 Pour une approche critique des modèles géométriques utilisés, $c f$. D. KNUTH, “Mathematical Typography”, Digital Typography (CSLI Lectures Notes), 78 (1999), p. 1965 ; Id., "The letter S ", id., p. 263-284 et Hermann ZAPF, "Typographie des caractères romains de la Renaissance". français (XIVe-XVII siècles), Paris, Éditions du Cercle de la Librairie, 2000, p. 181. 
l'attention sur cette mise au point. Un passage du fo VII a précise l'argument ${ }^{19}$. Lépigraphie romaine ne suffit pas à attester de l'origine romaine des lettres capitales. Renommer attiques les lettres antiques, c'est rendre aux Grecs le mérite de leur antériorité. La lettre capitale romaine n'est qu'un emprunt dont les Romains se sont attribué l'invention, conformément aux façons de pirates dont ils usaient envers les vaincus ${ }^{20}$. C'est grâce à l'abus de leur puissance militaire que les Romains ont usurpé le titre de civilisateurs ; au lieu de propagateurs de culture, ils se sont comportés en vandales. Rendre aux lettres leur véritable nom d'attique, c'est faire œuvre de justice. Mais Tory ne s'en tient pas là. De cette restitution de la Grèce, il découle qu'en en lieu et place de Rome, la civilisation grecque, que les Romains ont balayée pour se parer orgueilleusement de ses dépouilles, est la véritable mère de la France et des Français.

J'aborde ici le deuxième temps de cette étude : le lien de filiation directe qui unit la France à la Grèce antique. Le premier livre du Champ fleury passe en revue une série d'arguments attestant de l'origine grecque des Français. Tory invoque l'étymologie de certains mots français (paradis, ange, cygne) et la mention, dans le De origine et gestis Francorum compendium de Gaguin, publié en 1495, de lettres grecques envoyées à Constantinople par Louis le Pieux. Je ne détaillerai que le premier argument en faveur de la grécité des Français, un épisode mythologique, la fondation de Paris par Hercule en personne ${ }^{21}$.

$19 \mathrm{~F}^{\circ} \mathrm{VII}$ a : « Les dictes lettres Attiques sont deuement nommes Attiques, \& non Antiques, ne Romaines : pource que les Atheniens en ont vse auant que les Romains, ne homme de leur Italie, combien que lesdictz Romains \& Italiens en on faict leurs monstres en leurs sumptueux Palaix, \& Arcs triumphans, comme on peut encores veoir dedans Romme aux ruynes quon voit par cy \& par la enuyronnees de Canetieres en la plus grande partie de la dicte Romme. "

$20 \quad \mathrm{~F}^{\circ} \mathrm{V} \mathrm{b}$ : «Car luy (i. e. César) \& les Rommains estoient si gormans \& grans ambrasseurs de gloire, quilz ne vouloient seullement vaincre les Royaulmes \& Nations, mais en destruyssant Loix, Costumes, Vsagesn, \& toutes aultres bonnes choses, \& en demolissant Epitaphes \& sepulchres, ilz vouloient que leurs victoires $\&$ arrogances fussent mises en memoire par leurs lettres latines, cuydant exceder la langue Grecque, laquelle chose ilz nont peu faire en tant que la dicte langue Grecque est de lettres myeulx ordonnées, en sorte quelle est sans comparaison plus fertile, abundante, \& florissant que la leur Latine. » $\mathrm{F}^{\circ}$ Vla : « Les Grecs ont este Autheurs aux Latins en toute maniere de doctrine, tesmoing Priscian qui dict au premier livre de son art de Grammaire, au tiltre De accidentibus literae, quant il dit. Porro Greci quibus in omni doctrina authoribus vtimur. Les Grecz, dit il, sont noz autheurs en toute sorte de discipline.

Avant que le dit Cesar vint icy, \& y traynast la dicte langue Latine, les lettres Grecques y pouvoient estre, $\&$ de faict elles y estoient en cours, considere que long espace de temps, $\&$ grand nombre dans par auant, comme dit Baptiste Mantuan en ung de ses liures quil a faict en descriuant la vie de sainct Denis, quant Hercules alla outre Espaigne aux iardins des Hesperides, passa par ceste contree, \& quant il fut en lisse de ceste cite de Paris, il print grant plaisir a veioir le pais \& la riuiere de Seine, quil commenca a edifier, puis sen volant aller oultre a ses entreprinses, y laissa une bande $\&$ compaignie de ses gens darmes qui 
Le dieu qui est à l'origine de la fondation de la ville n'est autre qu'Hercule, en qui, conformément à l'autorité de Lucien, Geoffroy Tory reconnaissait l'Ogmios Gaulois, dieu dont la représentation figurait la puissance d'attraction que possède le logos 22 . Une gravure illustre l'allégorie de Lucien (fig. 6) ${ }^{23}$. Bien évidemment, sous la peau de lion du héros éloquent, on voit se dessiner la royale silhouette de François Ier, que ses contemporains célébraient à l'envi comme l'« Hercule Gaulois 24 ». Un tableau de l'allégorie qui figurait le roi en dieu de l'éloquence lors de l'entrée solennelle de la reine

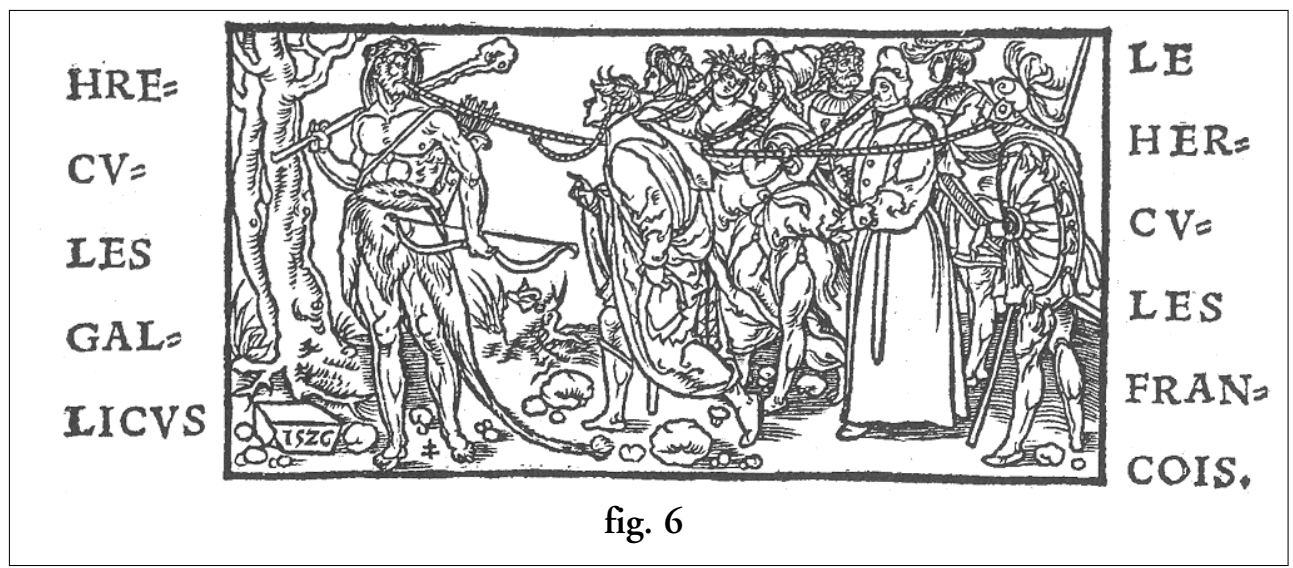

estoient appellez Parrhasians selon le nom de leur pais en Grece du coste Dasie, qui est nommee Parrhasia. Iceulx Parrhasians laisserent leur nom icy et en mutation de A en I les habitans de ceste dicte Cite ont este, \& sont encore dictz \& appellez Parrhisiens.

Doncques iceulx Parrhasians demourans icy, edifierent en la dicte isle, \& commancerent soubz bon $\&$ prospere horoscope ceste noble cite de Paris qui est auiourdhuy myelx que Athenes nestoient au temps passe, la fontaine de toutes sciences. La monnoie de toute vertu. Le theatre de nobles personages Lexcellence de bons esperitz. Le sanctuaire de deuotes ames. \& le tresor de tous biens".

Lucien Héraklès, 1-6. Cf. Marion Euskirchen, s. v. "Ogmios ”, Der Neue Pauly, 8, Stuttgart, J.B. Metzler, 2000, p. 1122 ; sur la continuité du motif, $c f$. Fr. LE RouX, " Le dieu celtique aux liens. De l'Ogmios de Lucien à l'Ogmios de Dürer ”, Ogam 12 (1960), p. 209-234 ; R.V. FreYr, "Raffaels Herakles Ogmios. Ein Paradigma zur Ikonologie des sprachlichen Wohlklanges ", in R.V. FreYr (éd.), Ainigma. Festschrift H. Rahn, Heidelberg, C. Winter, 1987, p. 35-75.

$\mathrm{F}^{\circ}$ III b. La composition de l'allégorie a été attribuée à Godefroy le Batave par M. ORTH, "The Triumphs of Petrarch illuminated by Godefroy le Batave (Arsenal, Ms. 6480) ", Gazette des Beaux-Arts 2e sem. (1984) [p. 197-206], p. 205 n. 11.

Cf. Anne-Marie LeCOQ, François Ier imaginaire. Symbolique et politique à l'aube de la Renaissance française, Paris, Macula, 1987, p. 425 et n. 75 p. 525. 
Éléonore à Rouen, en 1531, recourt à des termes fort proches de ceux de Lucien que cite Tory (f ${ }^{\circ}$ II b) ${ }^{25}$. Le motif fut réutilisé notamment par Henri II 26.

La cité fondée par Hercule-Ogmios reçoit des compagnons du dieu un nom trans-

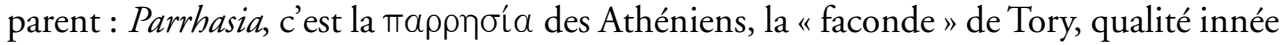
du peuple français ( $f^{\circ}$ II a) : "Nous sommes de nostre nature entre toutes les autres Nations, comme dict Pompone Mela, faconds, car il dit au Troisiesme liure de sa cosmographie, ou il parle des meurs des Francois, Habent tamen \& facundiam suam. Les Francois, dit il, sont faconds et beaux parleurs de nature ${ }^{27}$. » Fondée dans le sillage du dieu gaulois de l'éloquence, la future capitale de France reçoit un nom programmatique, puisqu'elle est destinée à devenir, sous la conduite de l'Hercule gaulois, la nouvelle Athènes, en partant à la reconquête de ses vertus innées. Tory n'écrit plus le nom de Paris qu'avec ce calembour orthographique, qui est un lieu commun au moins depuis Henri Estienne ${ }^{28}$. Lorsque l'auteur situe les Parrhasiens dans le paysage grec, il reste flou. Alors que les textes antiques en font des Arcadiens, Geoffroy Tory situe Parrhasia du côté de l'Asie ${ }^{29}$. Ce glissement topographique ne procède pas d'une erreur fortuite, mais d'une distorsion délibérée : détachés de l'Arcadie, les Parrhasiens deviennent des Ioniens, se rattachant de la sorte à la fois aux origines du dialecte athénien et

25 «En sa main droite tenoit en lieu de massue une lance entortillée d'ung serpent recouvert d'ung rameau de laurier signifiant que prudence en guerre est occasion de victoire... De sa bouche partoient quatre chesnettes, deux d'or et deux d'argent qui s'alloient attacher aux oreilles des personnages dessus nommez (Noblesse, Église, Conseil et Labeur). Mais elles estoient si tres laches que chacun les povoit juger ne servir de contraincte, ains qu'ilz estoient voluntairement tirez par l'eloquence du nouvel Hercule ", Les entrees de la reyne et de monseigneur daulphin/lieutenant general du roy \& gouverneur en ce pays de Normandie., Rouen, Raulin Gaultier, 1531.

Un arc de triomphe allait être élevé, le 16 juin 1549, à l'occasion de l'entrée de Henri II dans la capitale devant la porte Saint-Denis ; avec la devise Trahimur sequimusque volentes le monument serait surmonté d'une statue du dieu, avec les traits de François Ier et dont la bouche était reliée par quatre chaînes d'or à l'oreille de quatre personnages, figures des quatre états sociaux, $c f$. Anne-Marie LECOQ, François Ier imaginaire, n. 74 p. 525. Sur la notion de parrhesia, cf. G. SCARPAT, Parrhesia. Storia del termine e delle sue traduzioni in latino, Brescia, Paideia ed., 1964.

Henri Estienne adopte l'orthographe Parhisiis dans l'adresse du premier ouvrage qu'il édite dès 1502. C'est encore le cas dans sa Cosmographia de 1509, dont l'épître dédicatoire, rédigée par Geoffroy Tory, suit le même usage. Cf. Rabelais, Gargantua, ch. XVII : "Les Parisiens [...] dont estime Joaninus de Barranco, libro de copiositate reverentiarum, que sont dicts Parrhésiens en grécisme, c'est à dire fiers en parler "; dans le Discours non plus mélancolique que divers, paru à Poitiers en 1556 chez Enguilbert de Marnef : "il n’i a Parisiens qui ne sortent du Grec parrhesia, acause qu'aus femmes de Paris ne gela encores jamais le bec, qu'on sache " (p. 2).

$29 \mathrm{~F}^{\circ} \mathrm{VIa}$ : "Ses gens darmes qui estoient appeles Parrhasians selon le nom de leur pais en Grece du coste Dasie, qui est nomme Parrhasia. "Sur la localisation de Parrhasia, $c f$. E. Meyer, s. v. "Parrhasia, Parrhasioi (Arkadien) ", Real Enzyklopädie der Altertumswissehschaft Suppl.-Bd. 11, 1968, p. 1029-1033. 
au mythe d'Io développé plus haut. Les Parisiens sont ainsi non seulement parents des Athéniens, mais ils sont même, d'une certaine façon, issus d'une souche plus ancienne que la leur. C'est pourquoi, une fois retrouvé, le nom retrouvé de Paris appelle aussitôt, dans le style du panégyrique, une envolée eulogique.

Paris, c'est Athènes et la France est une Grèce. Cette équation est un préalable nécessaire à la perspective du Champ fleury. Tory veut encourager ses contemporains à produire une grammaire française, à doter la langue du royaume de cadres et de structures qui lui permettront de donner toute la mesure de son excellence. Cette tâche n'est pas impossible. L'histoire antique l'enseigne : avant d'être la Grèce, la Grèce a dû conquérir sa propre culture en se dotant de normes et de règles ${ }^{30}$. Il ne s'agit pas d'un simple parallélisme ou d'une analogie, mais d'une identité plénière : dans les dialectes mêmes qui divisent le royaume de France, Tory reconnaît la carte des dialectes grecs (pour accentuer la correspondance, il ajoute à leur inventaire la koinè) ${ }^{31}$.

L'appel à la normalisation du langage lancé par Geoffroy Tory n'est pas exclusif. Il embrasse tous les parlers de France. Dans les sections ultérieures du Champ fleury, l'auteur discute fréquemment des prononciations régionales et de leur rapport avec les usages graphiques en vigueur. C'est que, tous pareillement issus du grec, les dialectes français ont tous une dignité intrinsèque. En dépit de cette ouverture, la préférence de Tory va au parler de Paris. Dès le $\mathrm{F}^{\circ} \mathrm{Ib}$, il a vanté l'excellence que la langue française a

$30 \quad \mathrm{~F}^{\circ} \mathrm{IVb}$ : “ Sil est vray que toutes choses ont eu commancement, il est certain que la langue Grecque, semblablement la Latine ont este quelque temps incultes \& sans Reigle de Grammaire, comme est de present la nostre, mais les bons Anciens vertueux \& studieux ont prins peine, $\&$ mis diligence a les reduyre $\&$ mettre a certaine Reigle, pour en vser honnestement a escripre \& rediger les bonnes Sciences en memoire, au proufit $\&$ honneur du bien public."

$31 \mathrm{~F}^{\circ} \mathrm{IVb}$ : Quant Donatus, Servius, Priscianus, Diomedes, Phocas, Agrestius, Caper, Probus, \& les aultres bons Autheurs semblables furent venus, ilz la polyrent et mirent en si bonne ordre, que depuis a tousiours de bien en myeulx augmente en la perfection, si bien que les Romains, qui ont eu domination sus la plusgrande partie du monde, ont plus prospere, \& plus obtenu de victoires par leur langue que par leur lance. Pleust a Dieu que peussions ainsi faire, non pas pour estre Tyrans \& Roys sus tous, mais en ayant nostre langue bien reiglee, peussions rediger \& mettre bonne Science $\&$ Arts en memoire \& par escript. Ie voy que si nous voulons scavoir quelque Science, il la nous fault mandier $\&$ prendre quasi furtivement des Grecz \& des Latins, \& eulx nont que faire de nous, ne de ce que nous pouvons scavoir. Nostre langue est aussi facile a reigler \& mettre en bon ordre, que fut jadis la langue Grecque, en la quelle ya cinq diuersites de langage, qui sont la langue Attique, la Dorique, la Aeolique, la Ionique, \& la Comune, qui ont certaines differences entre elles en Declinaisons de noms, en Coniugations de verbes, en Orthographe, en Accentz \& en Pronunciation [...] Tout ainsi pourrions nous bien faire, de la langue de Court \& Parrhisienne, de la langue Picarde, de la Lionnoise, de la Lymosine, \& de la Prouuensalle. " 
déjà atteinte dans la capitale royale 32 . Le récit de la fondation par les Parrhasiens rappelle, un peu plus loin, que la capitale est, en quelque sorte, une France au carré, la nouvelle Athènes d'une nouvelle Grèce. Parmi les dialectes, l'attique occupe une position prééminente : Athènes est la Grèce de la Grèce.

Pour clore ce chapitre sur le paradigme grec dans le Champ fleury, je voudrais faire deux remarques : la première est que pour ses traces de gallomanie, son ardeur à prêcher à la fois la normalisation et la langue vernaculaire, il faut situer le livre de Geoffroy Tory sur une ligne qui va de Jean Lemaire de Belges (La Concorde des deux langages, c. 1510) au Devis de la langue française d'Abel Mathieu, paru en 1572, en passant, bien sûr, par la Défense et illustration de la langue française de Joachim du Bellay, publiée en 1549, et, dans une certaine mesure, le Traité de la conformité du langage françois avec le grec d'Henri Estienne, publié en 1565.

La seconde remarque relève moins de l'évidence, et concerne la dépréciation de Rome au profit de la Grèce. Dans l'abaissement des Romains, il faut voir probablement l'expression des tensions entre Valois et Habsbourg, entre François Ier et Charles-Quint, élu empereur le 28 juin 1519 et couronné roi des Romains à Aix-la-Chapelle le 23 octobre de l'année suivante. La première moitié des années 1520 voit une série de revers français face à l'Empire : la perte du Milanais (novembre 1521), la défection du connétable de Bourbon (1522-1523), les défaites de la Bicoque et de Pavie (1522 et 1525). Ainsi la France grecque est-elle le pendant de la Rome germanique, la véritable héritière de la véritable Antiquité. L'Hercule gaulois, champion de l'hellénisme, des Arts et des sciences, se dresse face à Rome l'usurpatrice et à César l'imposteur. En affirmant la supériorité de la culture grecque à l'empire romain, Tory emporte l'idée que le véritable rayonnement d'une cité tient davantage à sa production intellectuelle qu'au succès des armes. Grâce au détour par l'Antiquité, le Champ fleury contribue à restaurer l'image de François Ier : défait sur le terrain, l'Hercule gaulois est en réalité voué à la victoire 33 .

J'en viens au troisième thème : les résonances cosmologiques et anthropologiques du Champ fleury et leurs origines antiques. Le Champ fleury évoque à deux reprises une traduction de Horapollon due à Geoffroy Tory lui-même 34 . L'intérêt de Tory pour les

$32 \quad \mathrm{~F}^{\circ} \mathrm{I}$ b-II a : « Il est certain que le stile de Parlement, \& le langage de court sont très bons, mais encores pourroit on enrichir nostre dict langage par certaines belles Figures et Fleurs de rhetorique, tant en prose que autrement. "

Tory s'oppose à Alberti, tenant de la supériorité de Rome sur la Grèce, $c f$. Alberti, De re aedificatoria, VI, 3, dont on peut lire l'édition présentée et annotée par P. CAYE et Fr. CHOAY, L'art d'édifier, Paris, Seuil, 2004, p. 282.

$34 \mathrm{~F}^{\circ}$ XLIII a, signalé en manchette : «Ilz faisoient mille autres bonnes choses par Images, que vous porrez lire \& connoistre au .XXV. Chapistre des lecons antiques de Caelius Rhodiginus, \& plus aplain en Orus Apollo, qui les a redigees par escript, en ung volume que porres trouuer en Grec, si le y volez, \& en latin aussi, \& lequel iay translate en Francois, \& faict ung présent a ung myen seigneur et bon amy. " Une phrase analogue se retrouve au $\mathrm{f}^{\circ}$ LXXIII a ; quelques lignes plus bas, Tory cite Horapollon en latin avant d'en donner la traduction française. 
images égyptiennes, largement partagé par ses contemporains depuis l'édition aldine des Hieroglyphica (1505), vient renouveler le goût du XVIe siècle pour les emblèmes, ces images qui livrent au lecteur des figures sensibles de l'intelligible 35. Le Champ fleury montre une double tendance à voir dans les lettres des idéogrammes : dans le tracé du $\mathrm{A}$, Tory reconnait et surligne la silhouette d'une règle et d'un compas, outils souverains de l'art de tracer les lettres. Dans ce A - matrice de tous les autres caractères, selon l'un des deux mythes étiologiques rapportés au début du livre I - est ainsi lisible le secret de l'art lui-même (fig. 7) 36. Le A dit l'art de l'écriture. Plus significatif encore est, dans le Tiers livre, le commentaire de la lettre $\mathrm{Q}$, seul caractère à déborder du carré. Au livre précédent, Tory avait affirmé être le premier à proposer l'explication de cette singularité : le $\mathrm{Q}$, toujours accolé à un $\mathrm{V}$ qu'il amuit, est un $\mathrm{O}$ - figure de la perfection, suivie dans l'alphabet par le $\mathrm{P}$ de Prospérité - qui entoure de son bras le $\mathrm{V}$ de Vertu : « ceulx qui perseuerent es bonnes lettres [...] acquièrent des biens par leurs vertus " ( $f^{\circ}$ XII b et LIV b). Tory propose de lire dans la lettre $\mathrm{Q}$ une séquence invisible $(\mathrm{OP}) \mathrm{Q}(\mathrm{V})$ : les lettres s'appellent et se répondent et l'alphabet surgit au sein du caractère. Le tracé même du $\mathrm{Q}$ résume et figure tout le programme des belles lettres.

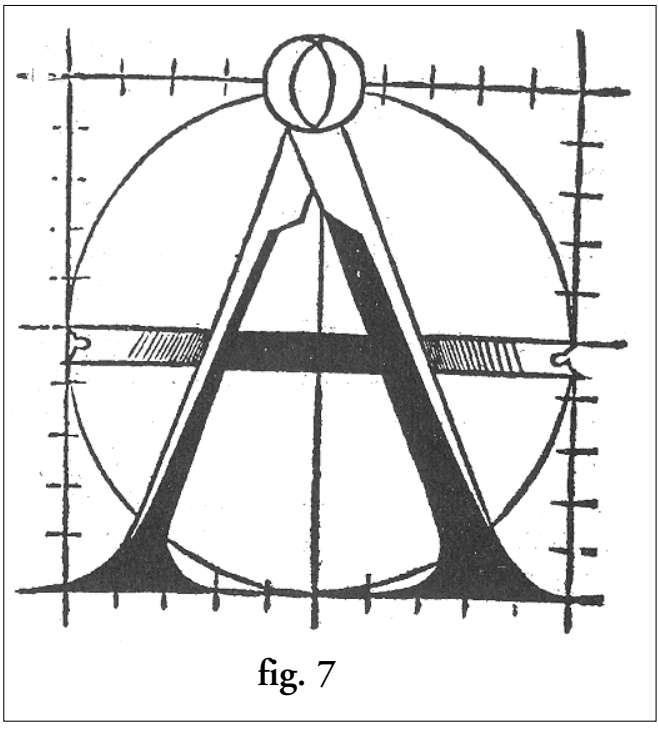

À ce langage des caractères, un autre modèle herméneutique se superpose, celui de traditions mathématiques issues de l'Antiquité. L'alphabet décline des formes, la droite et la courbe, en quatre types de tracés, vertical, horizontal, diagonal et « bouille » ou demicercle. Leur combinatoire laisse apparaittre trois formes de bases : le segment droit, l'angle et la courbe. Les considérations liminaires de Tory sur la double origine de l'alphabet soit I et $\mathrm{A}$, soit $\mathrm{I}$ et $\mathrm{O}$ - sont loin de constituer une digression, car elles proposent des étiologies de ces trois formes élémentaires et de leur combinaison. Les deux mythes confron-

Cf. A. Saunders, The Sixteenth Century French Emblem Book. A Decorative and Useful Genre, Genève, Droz, 1988. L'entreprise de Tory est contemporaine de celle d'Alciat, dont le Liber emblematum paraît à Augsbourg en 1531. Sur la diffusion de Horapollon, $c f$. R. AulotTe, “D'Égypte en France par l'Italie. Horapollon au XVIe siècle ”, in Mélanges à la mémoire de Franco Simone. France et Italie dans la cultre européenne, I, Genève, Slatkine, 1980 , p. 555-572.

$\mathrm{F}^{\circ}$ XXXIV b : «Entre tous les utilz manuels le Compas est le Roy \& la Reigle la Royne. Cest a dire les deux plus nobles $\&$ souverains, $\&$ soubz lesquelz, tous les autres utilz, $\&$ toutes choses bien ordonnees/ \& deument faictes sont raisonnables. " 
tent deux conceptions anciennes de la figure parfaite pour montrer que l'alphabet en réalise la synthèse. Le système graphique de Tory en arrive à proposer une lecture syncrétique d'Euclide et de Pythagore, Platon servant probablement de passeur de l'un à l'autre : Pour Euclide, le cercle est la figure parfaite ; pour Pythagore, c'est le triangle 37.

C'est que l'art d'écrire est une vraie science, attachée à la connaissance d'objets parfaits. La bonne écriture mène ainsi, littéralement, à la perfection. Cette idée n'est pas réductible à l'argumentaire promotionnel d'un technicien rédigeant un traité sur son art, réponse pleine d'émulation à l'éloge de l'architecture qu'Alberti, en digne héritier de Vitruve, avait récemment diffusé ; elle constitue une prise de position anthropologique. Les références de Tory sont à la fois géométriques et philosophiques; ses considérations sur la forme des lettres portent une théorie générale du monde et de la connaissance. La structure décimale du carré est en effet d'inspiration pythagoricienne. Le monde selon Pythagore est constitué de dix sphères emboîtées. Ainsi le carré à dix cases de côté est-il un graphique du monde 38 .

La combinatoire des figures met en évidence certains nombres : le un, lisible dans la droite et dans le point, le deux, correspondant aux extrémités de toute ligne, le trois, que figure le triangle, le quatre du carré, les dix niveaux du carré et du monde, les vingttrois lettres de l'alphabet. À l'instar des formes élémentaires, que sont la droite, l'angle et la "bouille ", ces chiffres livrent les principes universels du réel et constituent le dénominateur commun de l'homme, de la maison et du cosmos ${ }^{39}$. Pour accentuer la correspondance mathématique, Tory prend soin de noter que l'alphabet, outre son usage sémantique, peut revêtir une valeur numérique ( $\mathrm{f}^{\circ} \mathrm{XXXI} b$ ).

Dans la grille décimale, la lettre et l'Homme sont interchangeables. L'axe du I est un pivot de symétrie de part et d'autre duquel le visage de l'homme se dessine dans la quadrature. S'il est vertical, c'est pour souligner que la nature de l'homme fait tendre sa pensée vers le ciel ${ }^{40}$. L'homme est debout et sa verticalité permet que s'inscrive en

37 La figure du cercle est également développée par Vitruve, De architectura, III, 1. Sur le problème, chez Platon, de l'inscription du triangle dans le cercle, cf. Ménon, 86-87 a. Sur Tory et Euclide en particulier, $c f$. M. Cole, "Eine geometrische Wanderung durch die Renaissance : Gutenberg, Tory und Vignola übersetzen Euclides”, Gutenberg-Jahrbuch 67 (1992), p. 190-97.

On rapprochera la grille décimale de Tory du carré magique chez Agrippa de Nettesheim, qui constitue une figure du microcosme et révèle la permutabilité de la langue, des chiffres et des figures géométriques, $c f$. P. BÉHAR, Les langues occultes de la Renaissance. Essai sur la crise intellectuelle au XVI siècle, Paris, Desjonquères, 1996, p. 54-55. Cf. M. Sauret, J. Leblanc, "L'art de la proportion des lettres dans le Champ fleury: iconicité et narrativité", Texte 21-22 (1997), p. 97-106.

$\mathrm{F}^{\circ} \mathrm{XXI}$ a $:$ "Ie dis encores dauantage, que le I. qui est droict en ligne perpendiculaire ainsi assiz entre les deux yeulx, nous signifie que nous doibuons auoir le visage esleue enuers le ciel pour recognoistre nostre createur, \& pour contempler les grands biens \& la science quil nous donne. Et quil soit vray que Dieu veult quayons nostre contemplation enuers le ciel, il nous a donne la teste eleuee en sus, et aux bestes baissee en bas. " 
lui la hiérarchie du monde. Sur la grille du $\mathrm{O}$, dans le cercle qui est la figure géométrique parfaite, vient se poser un visage qui est à lui seul le microcosme du corps ${ }^{41}$. Les orifices du visage de l'homme, organes de perception et de connaissance, correspondent ainsi aux modalités des lettres ${ }^{42}$. Une troisième figure combine le $\mathrm{I}$ et le $\mathrm{O}$, pour former un $\Phi$ grec. Tory ne livre pas d'explication claire de cette figure dans le développement qui suit, mais note : « Les Grecs de ces deux lettres I \& O ainsi logees lune sus lautre comme les voyez en la ia dicte figure, ont faict une autre lettre quilz appellent Phi [...]. Il semble que nostre ditte figure soit ung rebus \& chose Hieroglyphyque, $\&$ que ie laye faicte pour faire resuer et muser les musards, mais tout bien considere, non est ». Il y a là une sorte d'énigme, dans laquelle je propose de voir une image de la philosophie : « Iay faict lesdites deux lettres I. \& O. \& la face humaine ensemble, pour touiours mieulx venir a la consommee perfection de noz lettres Attiques, qui sont XXIII. en nombre ». La justification de la figure est une tautologie. Dans le $\Phi$ qui s'imprime sur le visage, le lecteur retrouve à la fois, l'alpha et l'omega : le début - les deux lettres matricielles de l'alphabet - et la fin - la connaissance parfaite - (fo XXII a).

Lomniprésence du corps fait de la proportionnalité universelle de la figure humaine, inspirée du canon de Vitruve (III, 1), un leitmotiv. Du bas vers le haut, à chacun des organes de l'homme letre sont associés des arts libéraux. Cette correspondance reprend la mélothésie des Anciens ${ }^{43}$, projection à échelle réduite des planètes ou des signes du zodiaque dans les parties du corps humain, Tory présente un univers harmonique, où un système de connaissances s'incarne dans des corps physiques ou dans les abstractions géométriques mises en dessin que sont les caractères d'imprimerie. Le corps lui-même est un alphabet, dans lequel les lettres sont semées en désordre : " leur nature et vertu veult quelles soient meslees les unes aux autres ». Dans leur assemblage apparemment sans ordre, sur le mode de la marqueterie ou de la mosäque, les

$41 \quad \mathrm{~F}^{\circ}$ XXI b : « Aussi la teste humaine a en elle plus de sensualite $\&$ deficace que nulle autre partie du corps, entendu quelle a en elle sept conduyts \& origines desper $<\mathrm{i}>\mathrm{t}$ vital, en signification des sept arts liberaux [...] Les Oreilles sont, pour concevoir le nom des lettres. Les Yeulx, pour les cognoistre \& discerner. Les Narines pour armoniser la voix, \& le son en lesperant. Et la Bonche pour les pronuncer selon leur accent, leur ton, et leur distinction."

42 Une certaine capacité d'exercer les fonctions de la parole est reconnue à l'écrit. La tentation serait forte d'assimiler ce point de vue à celui d'un grammairien médiéval, habitué à distinguer entre les accidents de la lettre (nom, prononciation, tracé), comme le fait, par exemple, la Summa grammatica de Johannes Dacus (1280). Mais il y a, chez Tory, des échos assourdis d'un néoplatonisme éclectique.

Sur la mélothésie, $c f$. O. Ricoux, " Homo astrologicus : la mélothésie chez les astronomes latins ", in Ph. Moreau (éd.), Corps romains, Grenoble, Jérôme Millon (collection Horos), 2002, p. 201-223. 
vertus et grâces, les arts et les sciences, "rendent \& font ung œuvre tresbel \& parfaict 44 ». Le terme même de corps autorise toutes les permutations : l'organisme de l'homme letre est formé d'un assemblage de caractères. Le corps peut devenir lui-même un livre, quand les Éphésiens tracent sur leur propre peau des caractères en guise de talismans ( $\left.f^{\circ} \mathrm{XXXI}\right)$; inversement, le mot " corps » désigne l'unité de mesure de la lettre ( $f^{\circ} \mathrm{XI} b$ ) et le tracé des caractères est établi d'après le canon du corps humain.

Les capitales attiques de Tory sont ainsi des miniatures dans lesquelles se retrouvent toutes les échelles du monde. Miniatures du corps humain, les lettres sont aussi des figures de la maison, voire de l'architecture monumentale : la disposition du L indique comment situer les chambres d'un bâtiment par rapport au nord et au midi, et Tory lit dans les monuments romains des caractères gigantesques, projetés en trois dimensions ${ }^{45}$. Pour construire le carré décimal, sous-jacent à toutes les figures et dans les subdivisions duquel viendront s'inscrire les Muses ou les Arts libéraux ( $f^{\circ}$ XIIIIa), il faut tracer deux axes perpendiculaires, aussitôt transformés en signe de croix. Il ne s'agit pas là d'une profession de piété gratuite. Tory invoque à plusieurs reprises la croix et le nom de Jésus ${ }^{46}$. Tory relève ailleurs le lien entre la silhouette humaine ( $f^{\circ} \mathrm{XXXI}$ a) ou les trois points du triangle et la Trinité chrétienne, mais cette identification est associée à une série de références empruntées à l'Antiquité païenne ( $\left.\mathrm{f}^{\circ} \mathrm{Xa}\right)$. Ici, l'invocation place toutes les figures suivantes sous le signe du divin. La croix est à la fois le signe de la rédemption, la combinaison élémentaire de tracés simples qui est à la base de tout l'art typographique et un repère géométrique permettant de bâtir des figures justes. La géométrie des caractères s'articule simultanément avec l'élévation spirituelle et avec les quatre points cardinaux, soulignant la portée cosmique du carré décimal ${ }^{47}$. Même si

$44 \quad \mathrm{~F}^{\circ}$ XXIII a. Cette conception ne resta pas lettre morte. Elle se retrouve, dans le chapitre VI du Gargantua de Rabelais, dont la familiarité avec le monde de Geoffroy Tory est grande. Outre le passages cités supra n. 28, cf. Pantagruel II, 6 et M. PFISTER, " Parodie der französischen Gelehrtensprache bei Geoffroy Tory und François Rabelais ”, in Kl. Heitmann, E. Schroeter (éd.), Renatae litterae. Studien zum Nachleben der Antike und zur europäischen Renaissance (Festschift August Buck), Francfort a/M, Athenaeum, 1973, p. 193-205. Rabelais reprend le rébus du f XLIIa ( $G$ grand $a$ petit), $c f$. M. SAURET, "Quelques exemples de l'importance du morcellement des lettres et de leur montage dans Gargantua", Sborník prací folozofické fakulty Brnùnské university /Studia minora Facultatis philosophicae universitatis Brunensis, 17 (1997), p. 41-52.

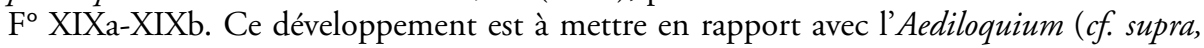
n. 8) ; cf. M. GrünberG-DröGe, "Geoffroy Tory's Aediloquium \& Epitaphia ", in R. SCHnur et al. (éd.), Acta conventus neo-latini Hafniensis, Binghamton (Medieval \& Renaissance Texts \& Studies), 1994, p. 443-453. $\mathrm{F}^{\circ}$ XXXIa, XLIVb; $c$. en particulier l'invocation à Jésus à l'explicit, $\mathrm{f}^{\circ}$ LXXIX.

$F^{\circ}$ XXXIIb : « Nous ferons doncques en la bonne heure, \& au nom de Dieu, tout premierement une croix [...] ». Dans la composition ternaire de la lettre A, que le mythe de la métamorphose de Hyacynthus associe au carré, Tory reconnaît au passage la Trinité chrétienne. Mais il la place sur le même plan que les usages rituels des nombres impairs chez les païens $\left(f^{\circ} \mathrm{Xa}\right)$. Lorsque les lettres sont présentées seules, sans la grille carrée, c'est 
elles héritent de traditions médiévales ${ }^{48}$, l'arithmologie du Champfleury et son obsession pour les structures cachées de l'alphabet prennent corps dans un contexte particulier : les Conclusions de Pic de la Mirandole avaient jadis attiré l'intérêt sur la signification secrète des lettres de la Bible ${ }^{49}$. Depuis une dizaine d'années au moins, François Ier montrait de d'intérêt pour les développements kabbalistiques sur l'écriture. En 1529, le roi éleva Jean Thénaud, un franciscain auteur de deux manuscrits sur la cabale, La saincte et très chrestienne cabale métrifiée, achevé en 1519 et le Traicté de la cabale, achevé en 1521, à la dignité d'abbé de Melinays. Thénaud entendait y montrer que " es escripts latins qui sont autant antiques que les hebraïques ou grecques est autant et plus de mistere qu'es susdictes $50 \%$. C'est probablement à ce contexte qu'il faut rapporter les spéculations pieuses et antiquaires de Tory sur la structure latente des lettres de son alphabet «attique ». Pour autant, Tory ne semble pas puiser directement ses conceptions dans la littérature hébraïque : lorsqu'il évoque des traditions exégétiques juives, c'est en faisant référence à Flavius Josèphe ${ }^{51}$. Il ne cite pas davantage les kabbalistes chrétiens, ne mentionnant Reuchlin que pour la grammaire « que le tresscauant Reuclin a faicte pour le tressingulier proufit des bons estudiens 52 ". Si le Champ fleury converge avec la pensée des kabbalistes sur les secrets des caractères, Tory met en ouvre une approche originale, issue de son intérêt pour les hiéroglyphes d'Horapollon.

qu'elles délivrent un enseignement particulier. Au fo XLIXb, l'image du L, la « lettre longue " illustre et moralise un passage de Plaute. Le L est figuré comme la transcription du corps humain tel qu'il se présente au monde, debout, à mi-route entre le plan horizontal de la terre et le plan vertical du soleil. La tête de l'être humain pointe vers le ciel, mais son ombre s'étire au sol. La culture complexe de Tory se révèle au passage : une mise au point philologique sur le sens controversé d'une expression de Plaute, donne forme à un emblème cosmologique.

On se reportera au petit livre récent de M. ZINK, Le Moyen Âge à la lettre. Un abécédaire médiéval, Paris, Tallandier, 2004.

Pic de la Mirandole, Conclusion XXXIII (1486), cf. Fr. ROUdAUlT, Le livre au XVIe siècle. Éléments de bibliologie matérielle et d'Histoire, Paris, Champion, 2003, p. 24.

50 La saincte et très chrestienne cabale métrifiée, BNF fr. 882); Traicté de la cabale, Arsenal, ms. 5061, cité fo LXXXXV) ; cf. Fr. SECRET, Les kabbalistes chrétiens de la Renaissance, Paris, Dunod, 1964, p. 153-156; sur la mode de la kabbale au XVIe siècle, cf. id., 306-322. Cl. G. Dubois, “ Corps de la lettre et sexe des nombres. Limagination de la forme dans le traité de Geoffroy Tory sur la Vraye proportion des lettres ", Revue des Sciences humaines 179.3 (1980), p. 77-91 ne s'intéresse pas directement aux convergences entre Tory et la kabbale.

$51 \quad \mathrm{~F}^{\circ} \mathrm{V}$ a : « Le susdict Iosephus a laisse par escript que les enfans de Adam inuenterent les figures \& caractheres des lettres, \& quilz les escripuirent en deux colomnes... ». Les notions d'hébreu que montre Tory remontaient probablement à sa collaboration avec Gilles de Gourmont, qui avait dès 1508 édité la grammaire hébraïque de François Tissard. 1506. 
La critique contemporaine a parfois allégué que le titre même de Champ Fleury relève du lieu commun pour désigner un type d'ouvrages riche en enseignements propres à orner l'esprit du lecteur ${ }^{53}$. Le champ fleuri offre à chacun de quoi composer son propre bouquet. Nous trouverions un vague équivalent avec nos " anthologies", nos " florilèges ", ce genre littéraire situé aux confins de la littérature savante et des écrits populaires, même si le champ fleuri suppose un type de lecture différent, une forme de butinage et d'appropriation du texte dont les manchettes, l'usage des annotations marginales et les recueils de loci gardent la trace ${ }^{54}$. Mais il ne s'agit là, je crois, que d'un effet de surface. Il faut prêter attention aux résurgences du titre au sein de l'ouvrage dans le premier envoi, à la fin du second envoi, mais aussi dans le commentaire de l'" homme letre » au livre second 55 . Cette répétition, à laquelle il faut ajouter une allusion indirecte au début du livre I 56, souligne l'implication progressive du titre dans l'argument de l'ouvrage : initialement présenté comme le choix d'une désignation à la fois ornementale, simple et mnémotechnique, le champ fleuri figure, lors de sa seconde apparition, l'univers de beauté et d'excellence auquel aboutira la purification de la langue et l'institution de règles pour la régir. Le motif résume ainsi une conception de la beauté à la fois esthétique et morale qui s'inspire, comme celle d'Alberti, de la notion cicéro-

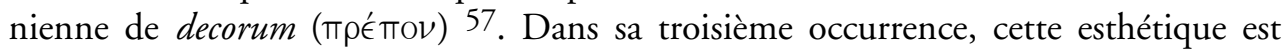

B.C. Bowen, “ Geoffroy Tory's Champ Fleury and Its Major Sources ”, p. 14.

Sur les manchettes de Tory, $c f$. en particulier Gérard Milhe Poutingon, " Les Notes marginales dans le Champfleury de Geoffroy Tory : des auxiliaires de lisibilité ”, in L'Espace de la note, J. DürrenmatT, A. Pfersmann (éd., introd.), Rennes, P.U. de Rennes, 2004, p. 67-82.

Envoi $A$ tous vrayz \& devotz amateurs de Bonnes Lettres: «Lettre Attique est si noble quelle veut estre en grande liberte, comme porrez veoir en ceste CEuvre que iay nomme Champ Fleury pour la grace et facilite du nom ". ; Envoi Aux lecteurs de ce Present Livre : "Par ainsi on trouuera que peu a peu on passera le chemin, si bien quon viendra aux grans Champs Poetiques et Rhetoriques plains de belles/ bonnes/ \& odoriferantes fleurs de parler \& dire honnestement $\&$ facillement tout ce quon vouldra "; f XXIII : " Nous voyons au prim temps que la beaulte dun pre $\&$ dun iardin est en la diuersite $\&$ multitude assemblee de diverses belles herbes $\&$ fleurs, qui de leur odeur rendent une suauite digne quasi destre appellee $\&$ ditte chose diuine $\&$ digne destre immortelle. »

"Les Ambassadeurs des Troiens ont leur voix toute florissante, \& leur oraison est dicte Lirioessa. Liria, en Grec, il men souuient bien, sont fleurs " (f IIIa).

$\mathrm{F}^{\circ} \mathrm{XXII} \mathrm{b}$ : «Car nos bons peres anciens ont este si vertueux en leurs speculations quilz ont volu secretement entendre que lhomme parfaict est celluy en qui les bonnes lettres $\&$ Sciences sont insinuees \& intimees si nien quen tous endroicts \& mouuemens de son corps est garny du bon mot que Cicero au XXXV. Chapitre du premier liure de ses Offices, \& au commancemant De Oratore Ad Brutum dict \& appelle en Grec. $\pi \rho \in \pi \circ \nu$ $\&$ en latin Decorum. Qui vaut autant a dire en nostre langage Francois decent \& conuenable en toutes ses actions...". 
développée : l'image du champ fleuri évoque la beauté naturelle du corps humain, dont les parties correspondent à des lettres assemblées sans respecter l'ordre alphabétique ; ces lettres mêmes correspondent à des qualités - arts, sciences et vertus -, que le corps humain montre associées dans le désordre. Cultivées, ces belles lettres et ces bonnes vertus feront du corps un jardin raisonné à la règle et au compas. Le développement de l'ouvrage constitue de la sorte une longue glose du titre, qui n'a plus rien d'un simple lieu commun.

Abstraction faite de ses gloses internes au Champ Fleury, un tel titre, défini par un libraire helléniste, fait écho à un passage fameux du Phèdre de Platon, où Socrate évoque les « jardins en caractères d'écriture " que l'homme sage ne saurait travailler que pour son divertissement et pour conserver ou transmettre sa mémoire ${ }^{58}$. Le parallélisme avec le texte platonicien est discrètement souligné par l'incipit, qui ancre le projet du Champ fleury dans la mémoire personnelle de son auteur ${ }^{59}$. Une marque laissée dans l'esprit de Tory par une expérience technique - un dessin réalisé pour un prestigieux commanditaire -, resurgit à la faveur des révolutions de la mémoire provoquées par une rêverie digestive. Il n'est pas fortuit que le souvenir surgisse de phénomènes physiologiques ; l'impression mémorielle de la lettre se forme, pour ainsi dire, en donnant figure aux mouvements de l'organisme de Tory. Ce n'est ici qu'une variation liminaire sur le thème des rapports entre caractère écrit et le corps humain, qui parcourt tout le Champfleury. Mais elle fonde le projet du livre et sa matière dans une mémoire doublement personnelle et apporte aux planches qui présentent l'homme lettre la

58 Platon, Phèdre, 276c-d (je traduis) : «- Socr. : Or, de l'homme qui détient la science du juste, celle du beau, celle du bon, devons-nous dire que, pour les semences qui sont les siennes, il a moins d'intelligence que n'en a le cultivateur ? - Phè. : Pas le moins du monde, en vérité ! - Socr. : Il ne sera donc pas sérieux quand il ira les écrire sur de l'eau, en les semant dans une eau noire, au moyen d'un roseau, avec des discours qui, impuissants, par le discours, à se porter secours à eux-mêmes, sont d'autre part impuissants à enseigner comme il faut la vérité. — Phè. Il n'est du moins pas vraisemblable qu'il le fasse!

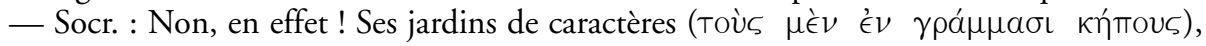
il les ensemencera, il les écrira, bien plutôt, pour se divertir. Quand, d'ailleurs, il lui arrive

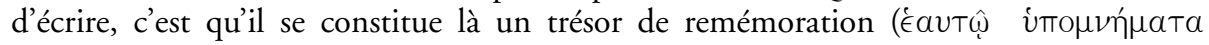
$\theta \eta \sigma a v p ı(o ́ \mu \in \nu 0 \zeta)$ et touche à la vieillesse oublieuse ; comme pour quiconque s'engage à

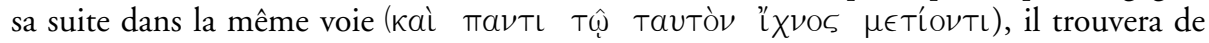
l'agrément à regarder croître ces fragiles jardins ». Dès avant 1421, le Phèdre avait été traduit à Florence par Leonardo Bruni.

$\mathrm{F}^{\circ} \mathrm{I}$ a : « Le matin du iour de la feste aux Roys, apres auoir prins mon sommeil \& repos, $\& \mathrm{q}<\mathrm{uand}>$ mon estomac de sa legiere $\&$ ioyeuse viande auoit faict sa facile concoction, que lon comptoit M.D.XXIII. me pris a fantasier en mon lict, \& mouuoir la roue de ma memoire/ pensant a mille petites fantasies, tant serieuses que ioyeuses, entre lesquelles me souuint de quelque lettre Antique que iauoys nagueres faicte pour la maison de mon seigneur le tresorier des guerres maistre Iehan groslier Conseiller \& Secretaire du Roy nostre sire, amateur de bonnes lettres, $\&$ de tous personnages sauans, desquelz aussi est tresfame et extime tant de la que deca des monts. " 
caution de l'expérience vécue. Ainsi faut-il voir, dans le champ fleuri de Geoffroy Tory, un art de faire épanouir son petit jardin de caractères, à la fois un manuel de jardinage et une collection de semences à l'usage du lecteur. Le livre, en tout et dans ses atomes que sont les caractères, est à la fois un instrument de connaissance et une figure du monde.

Le caractère hybride du texte, partagé entre des recommandations techniques sur l'art de bâtir le tracé des caractères à la règle et au compas et une entreprise de défense et illustration de la langue française, tient bien sûr aux usages de composition des humanistes français au début du XVI siècle. Cet aspect esthétique de l'œuvre est important et explique, en partie, sa postérité. Mais il réalise aussi un projet de mémoire et de diffusion du savoir directement inspiré par l'autorité de Platon, un système du monde dans lequel, aussi bien que l'esprit, et parce qu'elle en est la forme, la lettre vivifie.

\begin{tabular}{l} 
PIERRE CORDIER \\
\hline ERASME \\
Pavillon de la recherche \\
Université de Toulouse II-Le Mirail \\
5, allées Antonio Machado \\
31100 Toulouse \\
pierre.cordier@univ-tlse2.fr
\end{tabular}

